\title{
Correction to: Rel leaves of the Arnoux-Yoccoz surfaces
}

\author{
W. Patrick Hooper ${ }^{1}$ - Barak Weiss ${ }^{2}$
}

Published online: 11 August 2021

(c) Springer Nature Switzerland AG 2021

\section{Correction to: Sel. Math. New Ser. (2018) 24:875-934 https://doi.org/10.1007/s00029-017-0367-x}

Our arguments in $\S 6$ of the paper [3] contain an error. In this note we explain the error and how to fix it. We are grateful to Florent Ygouf for both pointing out the mistake, and for indicating the correct argument included below. All of the results stated in the introduction of the paper remain valid, as a consequence of the amended argument which will be given below. In the recent preprint [4], Ygouf proves related results about existence of dense rel leaves in other loci.

\subsection{The error}

The second assertion of Proposition 6.6 is wrong. Namely, the tangent space to $\mathcal{O}(x)$ is not a $\mathbb{Q}$-space. This affects the validity of Lemma 6.7: while it is true that $T_{r}$ and $\mathcal{O}_{r}$ are g-dimensional, it is not true that $T_{r}$ is a $\mathbb{Q}$-subspace. The rationality of $T_{r}$ is used once in the paper, in the proof of Theorem 6.9, at the end of the second paragraph of the proof.

\subsection{The area form}

Let $\langle$,$\rangle be the \mathbb{R}$-valued anti-symmetric bilinear form on $H^{1}(S ; \mathbb{R})$ defined by $\left\langle\beta_{1}, \beta_{2}\right\rangle=\int_{S} \beta_{1} \wedge \beta_{2}$. We extend this to a bilinear form on $H^{1}(S, \Sigma ; \mathbb{R})$ using the restriction map Res : $H^{1}(S, \Sigma ; \mathbb{R}) \rightarrow H^{1}(S ; \mathbb{R})$ by defining

The original article can be found online at https://doi.org/10.1007/s00029-017-0367-x.

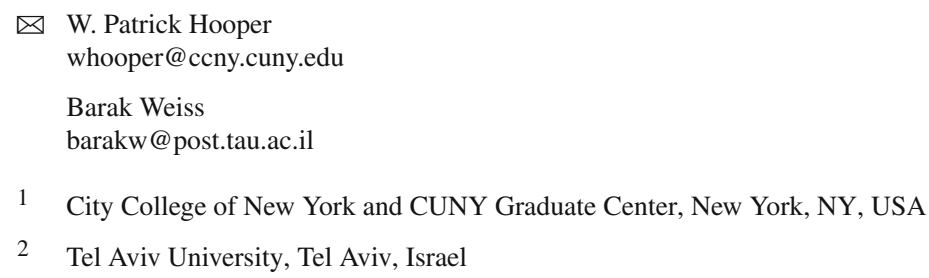


$\Theta: H^{1}(S, \Sigma ; \mathbb{R}) \times H^{1}(S, \Sigma ; \mathbb{R}) \rightarrow \mathbb{R} ; \quad \Theta\left(\eta_{1}, \eta_{2}\right)=\left\langle\operatorname{Res}\left(\eta_{1}\right), \operatorname{Res}\left(\eta_{2}\right)\right\rangle$

This form can be used to compute the flat area of a surface, namely, in the notation of [3],

$$
\operatorname{Area}\left(x^{\prime}\right)=\Theta\left(\operatorname{hol}_{x}\left(x^{\prime}\right), \operatorname{hol}_{y}\left(x^{\prime}\right)\right)
$$

Since $\Theta$ is defined in purely topological terms, for any orientation-preserving homeomorphism $\phi:(S, \Sigma) \rightarrow(S, \Sigma)$ we have $\Theta\left(\phi^{*} \beta_{1}, \phi^{*} \beta_{2}\right)=\Theta\left(\beta_{1}, \beta_{2}\right)$. See [2, $\S 3.2-3.3]$ and $[1, \S 2.5]$ for more information.

\subsection{Correcting the proof of Theorem 6.9}

In $[3, \S 6.3], \varphi$ is the Arnoux-Yoccoz pseudo-Anosov homeomorphism, formally defined in [3, Definition 3.5]. Section 6.3 defines subspaces

$$
T_{r} \subset P \subset H^{1}\left(S, \Sigma ; \mathbb{R}^{2}\right) \text {. }
$$

By Lemma 6.7, $\operatorname{dim} T_{r}=$ g. By Corollary 6.8, the subspace $P$ is $\mathbf{g}+1$ dimensional, is $\varphi^{*}$-invariant, and $P_{1}=\operatorname{span}\left\{\left(\operatorname{hol}_{y}\left(x_{0}\right), 0\right)\right\}$ is the dominant eigenspace for the action of $\varphi^{*}$ on $P$. The subspace $P_{2} \subset P$ is defined to be the $\varphi^{*}$-invariant subspace complementary to $P_{1}$, and so $P_{2}$ has dimension $\mathbf{g}$. We need to justify the claim made in the second paragraph of the proof of Theorem 6.9, that $T_{r} \not \subset P_{2}$. Since $\Theta\left(\operatorname{hol}_{x}\left(x_{0}\right), \operatorname{hol}_{y}\left(x_{0}\right)\right)$ is equal to the area of the surface $x_{0}$, it is not 0 , and thus

$$
P_{2}=\operatorname{ker} F \text { for } F: P \rightarrow \mathbb{R}, \quad F(\beta, 0)=\Theta\left(\operatorname{hol}_{x}\left(x_{0}\right), \beta\right) .
$$

(Note that elements of $P$ have the form $(\beta, 0)$ with $\beta \in H^{1}(S, \Sigma ; \mathbb{R})$.)

Our proof proceeds by contradiction. Suppose $T_{r} \subset P_{2}$. Then by dimension considerations, $T_{r}=P_{2}$, and in particular, since $T_{r}$ is the tangent space to the torus $\mathcal{O}_{r} \subset \mathcal{H}$, $\Psi^{-1}\left(\mathcal{O}_{r}\right)$ is a subtorus of $\mathbb{R}^{\mathbf{g}+1} / \mathbb{Z}^{\mathbf{g}+1}$, where $\Psi$ is the map defined in (6.9). Let $\widetilde{\Psi}$ be the map on p. 923 . The tangent space to $\Psi^{-1}\left(\mathcal{O}_{r}\right)$ is then $D\left[\widetilde{\Psi}^{-1}\right]\left(T_{r}\right)$. Since this tangent space is parallel to the subtorus $\Psi^{-1}\left(\mathcal{O}_{r}\right)$ of $\mathbb{R}^{\mathbf{g}+1} / \mathbb{Z}^{\mathbf{g}+1}$, any normal vector to $D\left[\widetilde{\Psi}^{-1}\right]\left(T_{r}\right)$ must be proportional to a rational vector.

We claim that a normal vector to $D\left[\widetilde{\Psi}^{-1}\right]\left(T_{r}\right)$ is given by $\left(c_{0}^{2}, c_{1}^{2}, \ldots, c_{\mathbf{g}}^{2}\right)$, where $c_{0}, \ldots, c_{\mathbf{g}}$ denote the circumferences of the $\mathbf{g}+1$ horizontal cylinders $C_{0}, \ldots, C_{\mathbf{g}}$ making up the surface $x_{r}$. To see this, observe that the function $F \circ D \widetilde{\Psi}$ vanishes on $D\left[\widetilde{\Psi}^{-1}\right]\left(T_{r}\right)$. By Proposition 6.1 and (6.9), we have

$$
D \widetilde{\Psi}\left(t_{0}, \ldots, t_{\mathbf{g}}\right)=\left(t_{0} c_{0} C_{0}^{*}+\ldots+t_{\mathbf{g}} c_{\mathbf{g}} C_{\mathbf{g}}^{*}, 0\right)
$$


where $C_{i}^{*}$ is as in (2.5). Thus,

$$
F \circ D \tilde{\Psi}\left(t_{0}, \ldots, t_{\mathbf{g}}\right)=\Theta\left(\operatorname{hol}_{x}\left(x_{0}\right), \sum_{i=0}^{\mathbf{g}} t_{i} c_{i} C_{i}^{*}\right)
$$

As $x_{0}$ and $x_{r}$ differ by a vertical rel deformation, we have

$$
\operatorname{hol}_{x}\left(x_{0}\right)=\operatorname{hol}_{x}\left(x_{r}\right) .
$$

Now let $x^{\prime}$ be a deformation of the surface $x_{r}$ with each cylinder $C_{i}$ stretched or compressed vertically so that it has an arbitrary height $h_{i}>0$. Then we have $\operatorname{hol}_{x}\left(x^{\prime}\right)=\operatorname{hol}_{x}\left(x_{r}\right)$ and $\operatorname{hol}_{y}\left(x^{\prime}\right)=\sum_{i=0}^{\mathbf{g}} h_{i} C_{i}^{*}$. Using $(0.1)$ we see

$$
\Theta\left(\operatorname{hol}_{x}\left(x_{0}\right), \sum_{i=0}^{\mathbf{g}} h_{i} C_{i}^{*}\right)=\operatorname{Area}\left(x^{\prime}\right)=\sum_{i=0}^{\mathbf{g}} h_{i} c_{i} .
$$

Extending linearly, we conclude that

$$
F \circ D \widetilde{\Psi}\left(t_{0}, \ldots, t_{\mathbf{g}}\right)=\sum_{i=0}^{\mathbf{g}} t_{i} c_{i}^{2}
$$

holds for all choices of the $t_{i}$. Since we have seen that $T_{r}=\operatorname{ker} F$, this proves the claim.

We have shown that $\left(c_{0}^{2}, c_{1}^{2}, \ldots, c_{\mathbf{g}}^{2}\right)$ is proportional to a rational vector. On the other hand, by Theorem 3.7 we have $\frac{c_{1}}{c_{0}}=\alpha$, and so $\alpha^{2}=\frac{c_{1}^{2}}{c_{0}^{2}} \in \mathbb{Q}$. But, this contradicts that the fact that $\operatorname{dim}_{\mathbb{Q}} \mathbb{Q}(\alpha)=\mathbf{g} \geq 3$.

\section{References}

1. Bainbridge, M., Smillie, J., Weiss, B.: Dynamics of the horocycle flow on the eigenform loci in $\mathcal{H}(1,1)$, to appear in Memoirs of the AMS (2019). arXiv:1603.00808

2. Forni, G., Matheus, C.: Introduction to Teichmüller theory and its applications to dynamics of interval exchange transformations, flows on surfaces and billiards. J. Mod. Dyn. 8(3-4), 271-436 (2014)

3. Hooper, W.P., Weiss, B.: Rel leaves of the Arnoux-Yoccoz surfaces, with an appendix by Lior BarySoroker. Mark Shusterman and Umberto Zannier, Selecta Math. 24(2), 875-934 (2018)

4. Ygouf, F.: A criterion for density of the isoperiodic leaves in rank 1 affine-invariant orbifolds, preprint (2020). arXiv:2002.01186

Publisher's Note Springer Nature remains neutral with regard to jurisdictional claims in published maps and institutional affiliations. 